\title{
PROYECTOS ESTOCÁSTICOS ORIENTADOS A LA ACCIÓN: UNA PUERTA AL DESARROLLO SOSTENIBLE DESDE TEMPRANA EDAD
}

\author{
STOCHASTIC ACTION-ORIENTED PROJECTS: A GATEWAY \\ TO SUSTAINABLE DEVELOPMENT FROM AN EARLY AGE \\ PROJECTOS ESTOCÁSTICOS ORIENTADOS À ACÇÃO: UMA PORTA \\ AO DESENVOLVIMENTO SUSTENTÁVEL DESDE A MAIS TENRA IDADE
}

\author{
Claudia Vásquez \\ Pontificia Universidad Católica de Chile (PUC), Villarrica, Chile \\ Recibido: 30/04/2021 - Aceptado: 24/05/2021 - Publicado: 01/12/2021 \\ Remita cualquier duda sobre esta obra a: Claudia Vásquez. \\ Email:cavasque@uc.cl
}

\begin{abstract}
RESUMEN
Desde hace varias décadas nos vemos desafiados a construir un mundo mejor, que permita satisfacer las necesidades actuales sin comprometer los recursos a futuro. Esto trae consigo el desafío de incorporar la educación para el desarrollo sostenible en el aula escolar desde temprana edad, buscando favorecer el desarrollo de competencias de sostenibilidad desde los primeros niveles educativos. En este escenario, la Educación Matemática en general y la Educación Estocástica en particular presentan gran potencial para el desarrollo de tales competencias. Desde este enfoque, en la primera parte de este artículo se describe el vínculo entre los proyectos estocásticos orientados a la acción y la educación en sostenibilidad y, en la segunda, se presentan experiencias de aprendizaje que utilizan los proyectos estocásticos para la acción como una herramienta para formar en sostenibilidad y allanar el camino hacia la alfabetización estadística y probabilística desde temprana edad. Se concluye que, mediante estas experiencias, los estudiantes, además de iniciarse en la alfabetización estadística y probabilística, reflexionarán y desarrollarán competencias de sostenibilidad, así como tomar conciencia respecto a problemáticas diversas vinculadas a los objetivos de desarrollo sostenible y a generar acciones en pos de un futuro mejor para todos.
\end{abstract}

Palabras clave: Ciclo de investigación; Sostenibilidad; Alfabetización estadística.

\begin{abstract}
For several decades now, we have been challenged to build a better world that meets current needs without compromising future resources. This brings with it the challenge of incorporating education for sustainable development into the school classroom from an early age, with the aim of encouraging the development of
\end{abstract}


sustainability competences from the earliest educational levels. In this scenario, mathematics education in general and stochastic education in particular have great potential for the development of such competences. From this approach, the first part of this article describes the link between action-oriented stochastic projects and sustainability education and, in the second part, presents various learning experiences that use stochastic projects for action as a tool for training in sustainability and paving the way from an early age towards statistical and probabilistic literacy. It is concluded that, through these experiences, students will not only be introduced to statistical and probabilistic literacy, but will also reflect on and develop sustainability competences, become aware of various issues linked to the Sustainable Development Goals and generate actions for a better future for all.

Keywords: Research cycle; Sustainability; Statistical literacy.

\section{RESUMO}

Há várias décadas temos sido desafiados a construir um mundo melhor, que permita satisfazer as necessidades actuais sem comprometer os recursos a futuro. Isto traz consigo o desafio de incorporar a educação para o desenvolvimento sustentável na sala de aula desde tenra idade, buscando promover o desenvolvimento de competências de sustentabilidade a partir dos primeiros níveis de ensino. Neste cenário, a educação matemática em geral e a educação estocástica em particular têm um grande potencial para o desenvolvimento de tais competências. A partir desta abordagem, na primeira parte deste artigo descreve-se a ligação entre projectos estocásticos orientados à acção e a educação para a sustentabilidade e, na segunda, apresentam-se experiências de aprendizagem que utilizam projectos estocásticos para a acção como uma ferramenta para formar em sustentabilidade e preparar o caminho à alfabetização estatística e probabilística desde idade precoce. Conclui-se que, mediante essas experiências, os estudantes, além de se iniciarem na literacia estatística e probabilística, reflectirão e desenvolverão competências de sustentabilidade, bem como tomarão consciência no tocante a problemáticas diversas ligadas aos objectivos de desenvolvimento sustentável e à gerarão de acçôes em prol de um futuro melhor para todos.

Palavras-chave: Ciclo de investigação; Sustentabilidade; Literacia estatística.

\section{INTRODUCCIÓN}

El año 2020 fue un año que nos golpeó en distintos ámbitos a nivel mundial: sanitarios, económicos, sociales, medioambientales, etc. Sin embargo, al mismo tiempo, nos ha brindado la posibilidad de apreciar la importancia de saber manejar e interpretar los datos, para lo cual es necesario contar con conocimientos de estadística/probabilidad y ciudadanos alfabetizados estadística y probabilísticamente (Gal, 2002, 2005). En este sentido, hoy más que nunca cobra relevancia lo señalado por H. G. Wells a comienzos del siglo XX: “el comprender promedios, máximos y mínimos algún día será tan necesario para una ciudadanía eficiente como lo es hoy la habilidad de leer y escribir” (Wells, 1911/1903, p. 204). Estas ideas incluso nos hacen cuestionar si hemos actuado con lentitud, pues hoy nos vemos enfrentados a la urgente necesidad de contar con una Educación Estocástica (Batanero, 2019) que impulse un pensamiento crítico orientado a la acción y que contribuya a transformar el mundo actual.

En este sentido, no es desconocido el hecho de que a diario nos vemos enfrentados a una cantidad desmesurada de información, catalogada como una verdadera infodemia (WHO, 2020) que viene a remarcar no solo la importancia de otorgar sentido a la enseñanza de la estadística y la probabilidad, sino también de 
abordar su enseñanza desde temprana edad, lo que sin duda permitirá allanar el camino para un desarrollo gradual de la alfabetización estadística y probabilística (Vásquez et al., 2021). En esta misma dirección, Alsina (2017) plantea tres argumentos para incorporar el estudio de la estadística y la probabilidad en el currículo infantil: a) la importancia de garantizar una educación de alta calidad que se ajuste a los cambios sociales; b) la importancia de la estadística y probabilidad en el desarrollo integral de los niños; y c) la importancia de la alfabetización estadística y probabilística desde las primeras edades. Argumentos que cobran total sentido en el actual contexto de infodemia, y más aún, si consideramos que recientemente la Organización para la Cooperación y el Desarrollo Económicos (OCDE) reconoce la necesidad de avanzar en el desarrollo de habilidades y conocimientos que permitan formar ciudadanos capaces de "extraer información significativa de los datos, comprender qué significan los datos, incluyendo cómo leerlos de manera apropiada, extraer conclusiones, así como reconocer cuándo se utilizan de manera engañosa o inapropiada” (OCDE, 2019, p. 5). Por consiguiente, es necesario promover la Educación Estocástica desde temprana edad, en especial si consideramos que los niños desde pequeños desarrollan su comprensión del mundo por medio de un razonamiento causal y estadístico como parte del desarrollo de sus conocimientos científicos, matemáticos y sociales (Yurovsky et al., 2013).

Desde esta perspectiva, el contexto desde el cual provienen los datos cobra especial relevancia (Vásquez et al., 2018) pues, no en vano, en la Educación Estocástica "los datos no son sólo números, sino números en contexto" (Cobb \& Moore, 1997, p. 801) y no se puede pensar el análisis de los datos sin pensar sobre el contexto en el que se ofrecen (Bargagliotti et al., 2020; Hahn, 2014; Wild \& Pfannkuch, 1999), pues "lo que importa no son los datos, sino las respuestas y los conocimientos que buscamos en los datos" (Gal, 2019, p. 4). Por tanto, el contexto determina de qué forma y qué datos recoger, cómo analizarlos e interpretar los resultados que se obtienen (Weiland, 2019), dando lugar a una continua interrelación entre el problema estocástico y el contexto del problema (Wild \& Pfannkuch, 1999). Pero no se trata de una tarea sencilla, pues "el contexto no está presente automáticamente en el aula -tenemos que introducirlo" (Gal, 2019, p. 3), quedando como responsabilidad del profesorado la correcta selección de éste.

Pero ¿qué contextos utilizar?, ¿qué contextos son los más relevantes? Las respuestas pueden ser muy variadas. Sin embargo, un contexto acerca del cual resulta de vital importancia poder reflexionar es el desarrollo sostenible (CMMAD, 1987).

Desde hace ya algunos años el mundo (nuestra casa común) se encuentra en medio de una verdadera crisis, cuyos esfuerzos por superarla, hasta ahora, no han sido del todo exitosos. Por consiguiente, urge tomar medidas que permitan contar con ciudadanos alfabetizados en sostenibilidad, esto implica el desarrollo de "habilidades, actitudes, competencias, disposiciones y valores necesarios para sobrevivir y prosperar en las condiciones declinantes del mundo de manera a mitigar dicho declive en la medida de lo posible” (Stibbe, 2009, p. 2). Alcanzar este desafío requiere de un esfuerzo conjunto de diversas disciplinas, que convoquen a un trabajo interdisciplinario. Esto conlleva una manera diferente de afrontar la educación del siglo XXI e impone no solo la necesidad de contar con ciudadanos 
alfabetizados en sostenibilidad (Wals, 2015), desafiando al profesorado a cargo de educar hoy a los ciudadanos del mañana.

En este sentido, la Educación Matemática en general y la Educación Estocástica en particular presentan gran potencial en el desarrollo de competencias clave para la sostenibilidad, situándose como un conocimiento crucial que todo ciudadano puede utilizar para contribuir a desarrollar una mejor sociedad, tanto en lo económico, social y medioambiental (Vásquez \& García-Alonso, 2020). Cabe precisar que estas competencias deben ser desarrolladas por todos los estudiantes, a distintos niveles según la edad, y no reemplazan a las competencias específicas de las distintas áreas, como por ejemplo la matemática, sino que las comprenden y les permite un mayor alcance (Rychen, 2003).

Por tanto, es necesario reorientar la enseñanza de la estadística y la probabilidad en el aula escolar hacia una enseñanza contextualizada, que transite hacia una Educación Estocástica para educar en sostenibilidad (Vásquez, 2020), en la que "los conceptos estadísticos vinculados al contexto deben abordarse como construcciones sociales, siguiendo la estrategia sugerida por el aprendizaje estadístico orientado a los datos” (Gattuso \& Ottaviani, 2011, p. 124). Esto desafía a dejar de lado la algoritmización que en muchas ocasiones se observa en la enseñanza de la estadística y la probabilidad (Batanero \& Díaz, 2011; Vásquez et al., 2019) y enfocar su enseñanza hacia el desarrollo de competencias clave de sostenibilidad que, por su naturaleza, conllevan la resolución de problemas que abordan temáticas diversas, pues la educación para la sostenibilidad "no solo integra contenidos tales como el cambio climático, la pobreza y el consumo sostenible dentro de los planes de estudio, sino que también crea contextos de enseñanza y aprendizaje interactivos y centrados en el alumno” (UNESCO, 2017, p. 7). Por esta razón el análisis e interpretación de datos tiene mucho por aportar cuando se vinculan, por ejemplo, a temáticas relacionadas con los Objetivos de Desarrollo Sostenible (ODS) (UNESCO, 2017). Así, "la actual necesidad de desarrollar competencias para el desarrollo sostenible se constituye en un propósito para enseñar estadística y a su vez la estadística se convierte en un medio para formar en sostenibilidad" (Vásquez, 2021, p. 169). Por lo que, si queremos contar con ciudadanos alfabetizados desde este enfoque, se debe desarrollar desde temprana edad la motivación y capacidad para comprender, interpretar, evaluar críticamente y, cuando sea pertinente, expresar opiniones en cuanto a información cuantitativa y estadística.

En esta dirección, diversos resultados de investigación sugieren que el trabajo con proyectos estocásticos es una herramienta poderosa para alcanzar tal alfabetización, a la vez de proveer de un contexto a la enseñanza de la estadística y la probabilidad, haciéndola más relevante (Batanero \& Díaz, 2011). A la vez, a través del ciclo de investigación estadística (Wild \& Pfannkuch, 1999) es posible potenciar argumentos basados en datos o cuestiones relacionadas con la incertidumbre y el riesgo del mundo real, que lleve a los estudiantes a tomar decisiones para crear un mundo más sostenible. Sabemos que un elemento clave dentro de las actividades y procesos que tienen lugar en cada una de las etapas del ciclo de investigación estadística son las representaciones estadísticas pues, además de formar parte de las 
ideas estadísticas fundamentales (Burrill \& Biehler, 2011), permiten organizar y presentar visualmente los datos para así obtener una visualización rápida de patrones de variabilidad y tendencia, argumentar e incluso tomar decisiones. Por ende, es necesario prestar atención a la comprensión de tales representaciones, en especial, a la comprensión gráfica, entendida como "la habilidad de los lectores de derivar significado desde gráficos creados por otros o por ellos mismos” (Friel et al., 2001, p. 132). No obstante, la investigación respecto a los gráficos estadísticos y su enseñanza pone de manifiesto que ésta a menudo se centra en el proceso de construcción, dando poca atención a su interpretación (González et al., 2011) pese a que, al revisar diversos medios de comunicación, se observa que gran parte de la información se comunica a través de gráficos estadísticos. Así pues, en sintonía con lo anteriormente expuesto, es de gran importancia abordar la comprensión gráfica desde temprana edad, de manera tal de favorecer, por ejemplo, que los estudiantes puedan apreciar la riqueza de los gráficos estadísticos para organizar, representar y comunicar visualmente datos provenientes de contextos diversos; además de su potencial en el desarrollo de un pensamiento crítico.

Bajo esta mirada vinculada a la sostenibilidad, será necesario que todos los estudiantes durante su etapa escolar no solo tengan la oportunidad de adquirir una alfabetización estadística y probabilística, sino también adquirir conocimientos, competencias, valores y actitudes a través de una educación de calidad que fomente la reflexión y la acción en su entorno, en pos de contribuir a un desarrollo sostenible (Vásquez, 2020; Vásquez \& Alsina, 2021).

Es en este contexto que, a través del presente artículo, en primer lugar, se argumenta acerca de la importancia de abordar la Educación Estocástica con foco en sostenibilidad desde temprana edad, para luego presentar un conjunto de experiencias de aula que buscan promover este enfoque. Esto con el propósito de que por medio de tales experiencias se potencie el desarrollo no solo de un pensamiento crítico, sino de distintas competencias clave para la sostenibilidad que permitan "empoderar y equipar a las generaciones presentes y futuras para satisfacer sus necesidades mediante un enfoque equilibrado e integrado de las dimensiones económica, social y ambiental del desarrollo sostenible" (Leicht et al., 2018, p. 7).

\section{FUNDAMENTACIÓN TÉORICA}

Los campos de estudio que fundamentan teóricamente la propuesta presentada en este artículo son: la educación para el desarrollo sostenible, las conexiones entre la Educación Estocástica y la educación para el desarrollo sostenible y los gráficos estadísticos como una herramienta para educar en sostenibilidad.

\section{LA EDUCACIÓN PARA EL DESARROLLO SOSTENIBLE}

De acuerdo con el informe Brundtland de la Comisión Mundial del Medio Ambiente y del Desarrollo, "el desarrollo sostenible es un desarrollo que satisface las necesidades de las generaciones 
actuales sin comprometer la satisfacción de las necesidades de las generaciones futuras" (CMMAD, 1987, p. 24). Si bien el desarrollo sostenible representa un desafío de responsabilidad colectiva que nos convoca desde finales de los años 80, hoy la necesidad de promover una Educación para el Desarrollo Sostenible (EDS) es aún mayor, pues "la drástica transformación a escala planetaria debida a actividades humanas ha llevado a la Tierra a una nueva era geológica, el Antropoceno” (Casas et al., 2017, p. 114). En consecuencia, es necesario impulsar acciones prácticas para construir juntos un futuro mejor y avanzar así hacia una EDS que permita, para el año 2030,

Garantizar que todos los alumnos adquieran los conocimientos teóricos y prácticos necesarios para promover el desarrollo sostenible, entre otras cosas mediante la educación para el desarrollo sostenible y la adopción de estilos de vida sostenibles, los derechos bumanos, la igualdad de género, la promoción de una cultura de paz y no violencia, la ciudadania mundial y la valoración de la diversidad cultural y de la contribución de la cultura al desarrollo sostenible. (UNESCO, 2015, p. 20)

Para ello, la Organización de las Naciones Unidas (ONU) reconoce las dimensiones de acción económica, social y ambiental, a partir de las que se sugieren 17 ODS (Figura 1). Estos objetivos cubren una amplia variedad de temáticas que desafían a contar con una educación holística, integradora y transformadora que facilite que las generaciones actuales y futuras puedan alcanzar aprendizajes cognitivos, socioemocionales y conductuales específicos y, en especial, desarrollar competencias clave para la sostenibilidad (UNESCO, 2017).

\section{Figura 1}

Objetivos de Desarrollo Sostenible (UNESCO, 2017)
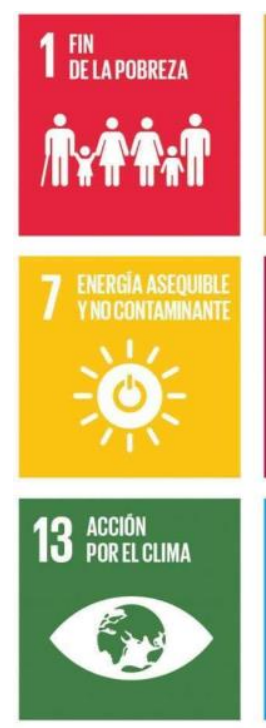
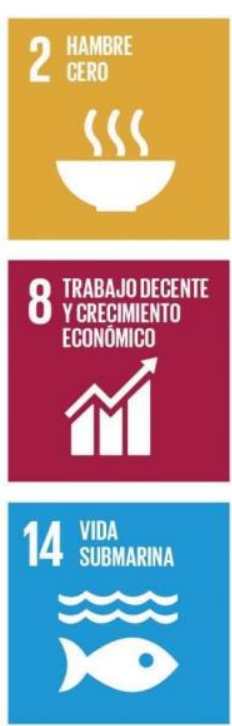
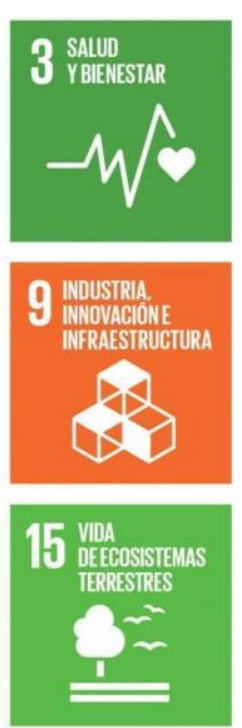
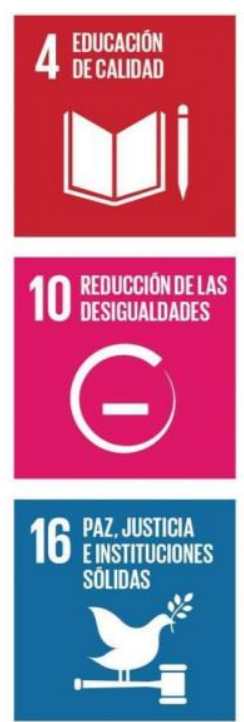
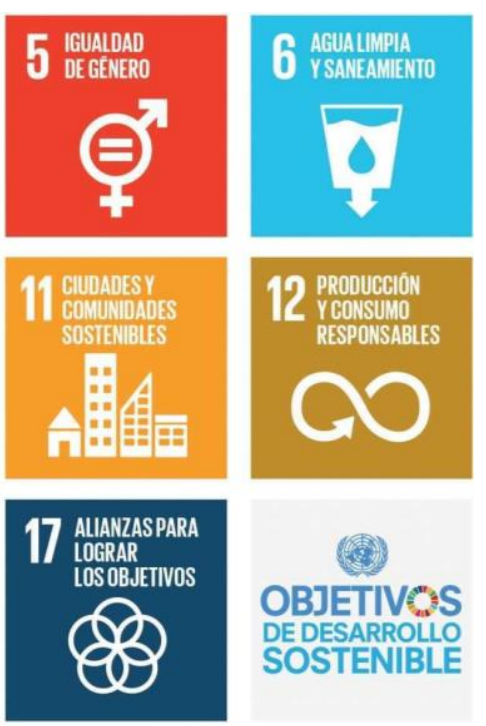

(69)

OBJETIVES DEDESARROLLO SOSTENIBLE 
Estas competencias "representan lo que los ciudadanos sostenibles necesitan específicamente para lidiar con los desafíos complejos de la actualidad. Son relevantes para todos los ODS y también permiten a los individuos vincular los distintos ODS entre sí” (UNESCO, 2017, p. 11). Así, de acuerdo con la UNESCO (2017, p. 10), se consideran las siguientes competencias clave para la sostenibilidad y las habilidades que incluyen:

1. Competencia de pensamiento sistémico: reconocer y comprender las relaciones; analizar los sistemas complejos; pensar cómo están integrados los sistemas dentro de los distintos dominios y escalas; y lidiar con la incertidumbre.

2. Competencia de anticipación: comprender y evaluar múltiples escenarios futuros; crear visiones propias de futuro; aplicar el principio de precaución; evaluar las consecuencias de las acciones; y lidiar con los riesgos y los cambios.

3. Competencia normativa: comprender y reflexionar sobre las normas y valores que subyacen en nuestras acciones; y negociar los valores, principios, objetivos y metas de sostenibilidad en un contexto de conflictos de intereses y concesiones mutuas, conocimiento incierto y contradicciones.

4. Competencia estratégica: desarrollar e implementar de forma colectiva acciones innovadoras que fomenten la sostenibilidad a nivel local y más allá.

5. Competencia de colaboración: aprender de otros; para comprender y respetar las necesidades, perspectivas y acciones de otros (empatía); para comprender, identificarse y ser sensibles con otros (liderazgo empático); abordar conflictos en grupo; y para facilitar la resolución de problemas colaborativa y participativa.

6. Competencia de pensamiento crítico: cuestionar normas, prácticas y opiniones; reflexionar sobre los valores, percepciones y acciones propias; y adoptar una postura en el discurso de la sostenibilidad.

7. Competencia de autoconciencia: reflexionar sobre el rol que cada uno tiene en la comunidad local y en la sociedad (mundial); evaluar de forma constante e impulsar las acciones que uno mismo realiza; y lidiar con los sentimientos y deseos personales.

8. Competencia integrada de resolución de problemas: aplicar distintos marcos de resolución de problemas a problemas de sostenibilidad complejos e idear opciones de solución equitativa que fomenten el desarrollo sostenible, integrando las competencias antes mencionadas.

Con este propósito, la UNESCO, a través de su programa de acción mundial para el desarrollo sostenible, establece cuatro enfoques a considerar al momento de diseñar e implementar experiencias de aprendizaje con foco en sostenibilidad: integral, crítico, transformador y contextual (Vásquez \& GarcíaAlonso, 2020).

Sin duda, el diseño e implementación de experiencias de aprendizaje que incorporen tales enfoques representan un reto que requiere adoptar perspectivas de enseñanza disciplinarias, 
interdisciplinarias y transdisciplinarias sobre temas de sostenibilidad, que converjan en una pedagogía transformadora orientada a la acción "que involucre a los alumnos en procesos de pensamiento y acción participativos, sistémicos, creativos e innovadores en el contexto de las comunidades locales y las vidas cotidianas de los alumnos” (UNESCO, 2017, p. 52).

\section{CONEXIONES ENTRE LA EDUCACIÓN ESTOCÁSTICA Y LA EDUCACIÓN} PARA EL DESARROLLO SOSTENIBLE

Ante este desafío, son diversos los autores que señalan la importancia de conectar la Educación Matemática con la sostenibilidad desde edades tempranas (Alsina \& Calabuig, 2019; Alsina \& Mulà, 2019; Joutsenlahti \& Perkkilä, 2019; Vásquez, 2020; Novo et al., 2020). Más concretamente, Vásquez (2020) posiciona a la Educación Estocástica como una herramienta para educar en sostenibilidad, puesto que la naturaleza de la educación estadística y probabilística "es inseparable de sus aplicaciones, y su justificación final es su utilidad en la resolución de problemas externos a la propia estadística” (Batanero \& Díaz, 2011, p. 21). Por tanto, es necesario enfocar la enseñanza de la estocástica a partir de contextos reales, adecuados a la edad y a la etapa escolar de los estudiantes, que tengan significado para ellos y que les permita avanzar hacia el aprendizaje de conceptos estocásticos, el empleo de técnicas de cálculo, mejorar sus capacidades de argumentación, formulación de conjeturas, reflexión y acción en torno a dicho contexto (Vásquez, 2020; Vásquez et al., 2018).

Lo anterior implica una manera diferente de pensar y de abordar la enseñanza de la estadística y la probabilidad en el aula escolar, "de manera tal que todas las personas tengan la oportunidad de constituirse, no solo como ciudadanos alfabetizados en estadística y probabilidad, sino también adquirir conocimientos, competencias, valores y actitudes con los que puedan contribuir al desarrollo sostenible” (Vásquez et al., 2021, p. 20). Donde la alfabetización estadística y probabilística es entendida como una competencia básica para evaluar datos en contextos diversos (Gal, 2002, 2005) y la Educación Estocástica para Educar en Sostenibilidad se concibe como la enseñanza de la estocástica a partir del abordaje de problemáticas provenientes de contextos reales y locales vinculados con los ODS, con el propósito de desarrollar competencias que empoderen al profesorado y, por ende, a los estudiantes para reflexionar y actuar sobre cuestiones vinculadas al desarrollo sostenible.

Esta doble aproximación permitirá, por un lado, que los estudiantes conozcan y tomen conciencia de la EDS y de los ODS y reflexionen respecto de qué pueden hacer para contribuir a alcanzarlos desde las propias acciones (tomar decisiones) y, por otro lado, otorgar sentido al aprendizaje de la estadística y la probabilidad, permitiendo así una mayor interiorización de lo aprendido (Vásquez, 2020; Vásquez \& García-Alonso, 2020). Pero asumiendo que no se trata sólo de que los estudiantes comprendan las distintas problemáticas, sino que es necesario ir más allá, de manera tal que los 
estudiantes tomen conciencia de estas problemáticas y actúen para avanzar hacia un mundo sostenible (UNESCO, 2017).

De acuerdo con Vásquez (2020), un aspecto importante a considerar para abordar la enseñanza desde este enfoque, es el trabajo con proyectos estocásticos (acordes a la edad de los estudiantes), pues además de mejorar la comprensión de los contenidos involucrados en el estudio de la estadística y la probabilidad, por medio de un aprendizaje más motivador y dotado de sentido, permiten favorecer el desarrollo de la alfabetización estadística y probabilística, al mejorar la percepción sobre su utilidad, contribuyendo de este modo a fomentar una actitud positiva hacia su estudio (e. g., Anasagasti \& Berciano, 2016; Batanero \& Díaz, 2011; Inzunza, 2017; Vásquez, 2020; Wild \& Pfannkuch, 1999).

En el trabajo con proyectos estocásticos, un aspecto clave es el contexto desde el cual provienen los datos con los que se trabajará, pues pueden ser datos recolectados por los propios estudiantes o bien datos provenientes de otras fuentes (bases de datos, periódicos, noticias, etc.). No obstante, es importante que los estudiantes trabajen con datos provenientes de contextos reales, que frecuentemente requieren de interpretaciones y razonamientos de alto nivel (Batanero et al., 2013); que desafíen a los estudiantes en la aplicación de sus conocimientos de estadística y probabilidad para resolver problemas procedentes de contextos reales y significativos, de manera similar a como lo hacen los estadísticos, siguiendo los pasos de un ciclo de investigación estadística (Figura 2) (Wild \& Pfannkuch, 1999). Un aspecto importante que resaltar es el potencial que presentan los proyectos estocásticos para adoptar perspectivas disciplinarias, interdisciplinarias y transdisciplinarias sobre temas de sostenibilidad.

\section{Figura 2}

Ciclo de investigación estadistica (Wild y Pfannkuch, 1999)

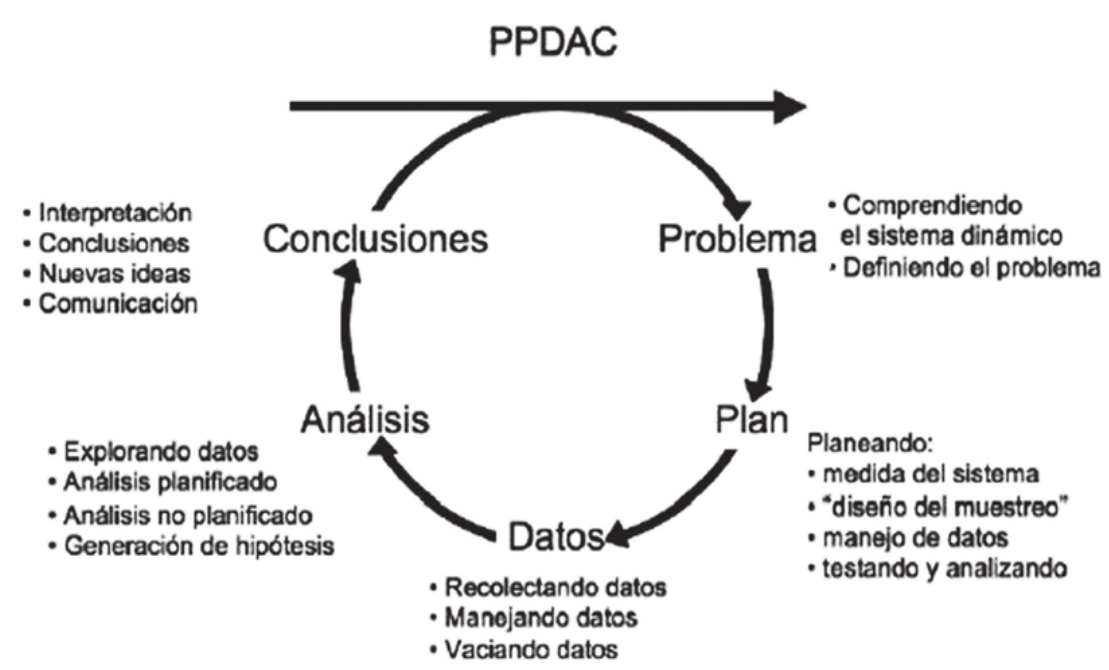

El abordar la enseñanza de la estocástica desde este enfoque no solo transforma el rol del profesor, sino también el de los estudiantes, permitiéndoles alcanzar un aprendizaje significativo a partir del trabajo en equipo (Anasagasti \& Berciano, 2016) al experimentar in situ el proceso de recolección y 
exploración de datos, favoreciendo así el desarrollo de la alfabetización estadística y probabilística (delMas, 2004). Por otra parte, permite apoyar el desarrollo de las competencias clave para la sostenibilidad a lo largo de las distintas etapas del ciclo de investigación (Figura 3), en especial, en aquella vinculada a las conclusiones del proyecto estocástico.

\section{Figura 3}

Conexiones entre el ciclo de investigación estadistica y educación para la sostenibilidad

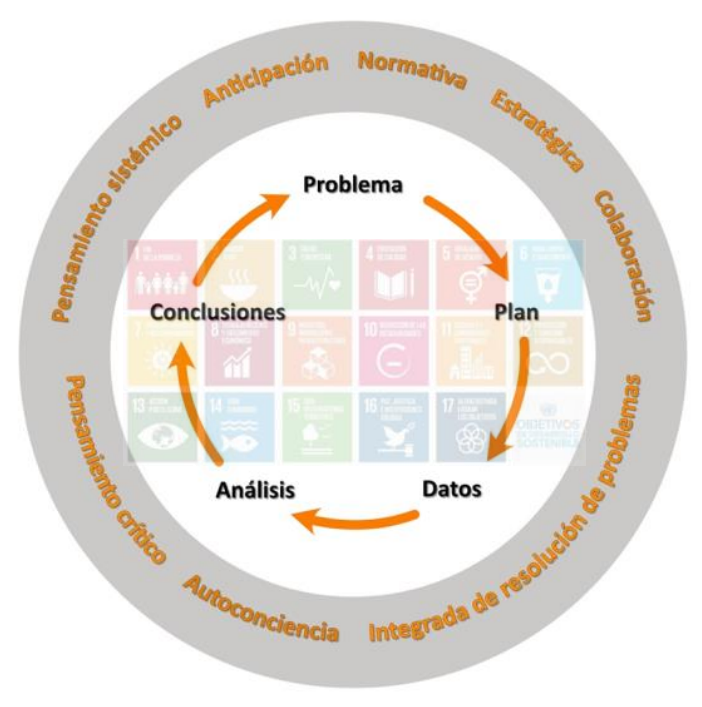

Así, el promover una enseñanza desde el enfoque de proyectos estocásticos orientados a la acción (siguiendo las etapas del ciclo de investigación estadística) en torno a problemáticas que provengan de contextos diversos vinculados con los ODS, que sean significativos y acordes a las edades de los estudiantes, favorecerá una enseñanza y aprendizaje en contexto, conduciendo a una educación orientada al desarrollo de ciudadanos alfabetizados en sostenibilidad.

Desde este enfoque, el rol del profesor en las distintas etapas del ciclo de investigación estadística es clave, pues debe favorecer que los estudiantes desarrollen las distintas competencias clave para la sostenibilidad.

\section{LOS GRÁFICOS ESTADÍSTICOS COMO UNA HERRAMIENTA PARA EDUCAR EN SOSTENIBILIDAD}

Dentro del trabajo con proyectos estocásticos es necesario prestar atención a la comprensión de las representaciones estadísticas, en especial, a la comprensión gráfica, pues todo ciudadano del siglo XXI debiera ser capaz de leer críticamente los gráficos estadísticos provenientes de diversos medios de comunicación. Cabe señalar que esto no solo conlleva una lectura literal de tales representaciones, sino también la capacidad para identificar tendencias, variabilidad, asociaciones de los datos y detectar posibles errores que pueden llevar a distorsionar la información representada (Arteaga et al., 2011). 
La comprensión de gráficos estadísticos se configura como un elemento clave en el desarrollo de la alfabetización estadística (Arteaga et al., 2016) y permitirá, a partir del trabajo con datos provenientes de contextos cercanos y significativos, que los estudiantes tomen conciencia sobre sus propias acciones en el entorno, de manera tal que tomen decisiones que modifiquen su actuar y, por ende, su estilo de vida hacia uno sostenible (Vásquez, 2021; Vásquez \& García-Alonso, 2020; Vásquez et al., 2021). Ahora bien, la lectura y comprensión de gráficos es un campo bastante explorado, ofreciendo desde hace varias décadas diversos análisis respecto de lo que implica su comprensión (e.g., Curcio, 1987; Friel et al., 2001; Shaughnessy et al., 1996). Esto ha llevado a proponer cuatro niveles de lectura de gráficos a partir de los trabajos de Curcio (1987), Friel et al. (2001) y Shaughnessy et al. (1996), que corresponden a: "leer los datos" (lectura literal del gráfico sin interpretar la información contenida en el mismo); "leer dentro de los datos" (interpretación e integración de los datos en el gráfico); "leer más allá de los datos" (predicciones e inferencias a partir de los datos sobre informaciones que no se reflejan directamente en el gráfico); y "leer detrás de los datos" (valorar críticamente el método de recogida de datos, su validez y fiabilidad, así como las posibilidades de extensión de las conclusiones). Esta gradualidad de niveles de lectura de gráficos servirá no solo de marco para formular preguntas en un contexto pedagógico, sino también para reflexionar en pos del desarrollo, por ejemplo, de un pensamiento crítico para la toma de decisiones, respecto de cuestiones vinculadas a problemáticas. Así pues, los estudiantes tendrían oportunidad de desarrollar la alfabetización estadística y probabilística en el aula escolar y, a la vez, adquirir conocimientos, competencias, valores y actitudes con los que puedan contribuir a un desarrollo sostenible (Vásquez, 2020).

Desde este prisma, y considerando la importancia de abordar la enseñanza de la estocástica a partir de contextos reales, en lo que sigue se presenta una selección de experiencias de aula que buscan promover la comprensión gráfica, así como el desarrollo de competencias para la sostenibilidad, desde el trabajo con proyectos estocásticos que abordan problemáticas vinculadas a distintos ODS, ajustadas a los niveles educativos de Educación Infantil y Educación Primaria.

\section{EXPERIENCIAS DE AULA PARA PROMOVER COMPETENCIAS PARA LA SOSTENIBILIDAD}

\section{EXPERIENCIA 1: ¿QUÉ QUIERES SER CUANDO SEAS GRANDE?}

- Edad:4-6 años.

- Contenidos implícitos: etapas del ciclo de investigación estadística, representaciones gráficas (gráficos concretos), frecuencia absoluta, comparación de frecuencias, variables cualitativas, categorías de una variable.

- Objetivo de desarrollo sostenible: ODS 5 - Igualdad de Género. 
- Competencias clave de sostenibilidad: pensamiento sistémico, anticipación, normativa, estratégica, colaboración, pensamiento crítico, integrada de resolución de problemas.

\section{DESCRIPCIÓN DE LA ACTIVIDAD}

Esta actividad forma parte de un conjunto de experiencias de aprendizaje que utilizan "el cubo para la sostenibilidad". Se trata de un cubo personalizado en el que cada niño coloca sus respuestas frente a distintas preguntas, por ejemplo: ¿cómo vienes a la escuela?, ¿cuántos vasos de agua consumes cada día?, ¿reciclan en tu casa?, ¿cuántas veces a la semana practicas deporte?, ¿cuál es tu snack preferido?, ¿qué quieres ser cuando grande?

Cada una de estas preguntas de investigación apunta a abordar ciertos temas en específico que son de interés para los estudiantes de estas edades, y que a la vez se vinculan con algunos de los 17 ODS.

En esta ocasión detallamos cómo se puede abordar el ODS 5 - Igualdad de Género a partir del desarrollo de un proyecto estocástico en torno a la pregunta de investigación ¿qué quieres ser cuando grande?

En primer lugar, el docente introduce este tema y plantea la pregunta anterior a sus estudiantes. Sin duda, las respuestas que surgirán serán muy variadas, y con muchas opciones de respuesta dado que es una pregunta de respuesta abierta. En dicho escenario una opción es trabajar con las distintas categorías de respuestas o bien acotar dichas categorías. Para ello, una estrategia puede ser que el profesor entregue a cada niño un cubo y un conjunto de láminas (Figura 4) que, en este caso, aluden a diversas profesiones u oficios que pueden ser de interés para los niños de estas edades (constructor/a, chef, garzón/a, doctor/a, profesor/a, heladero/a, policía, ejecutivo/a, cantante).

\section{Figura 4}

Selección de materiales y profesiones u oficios

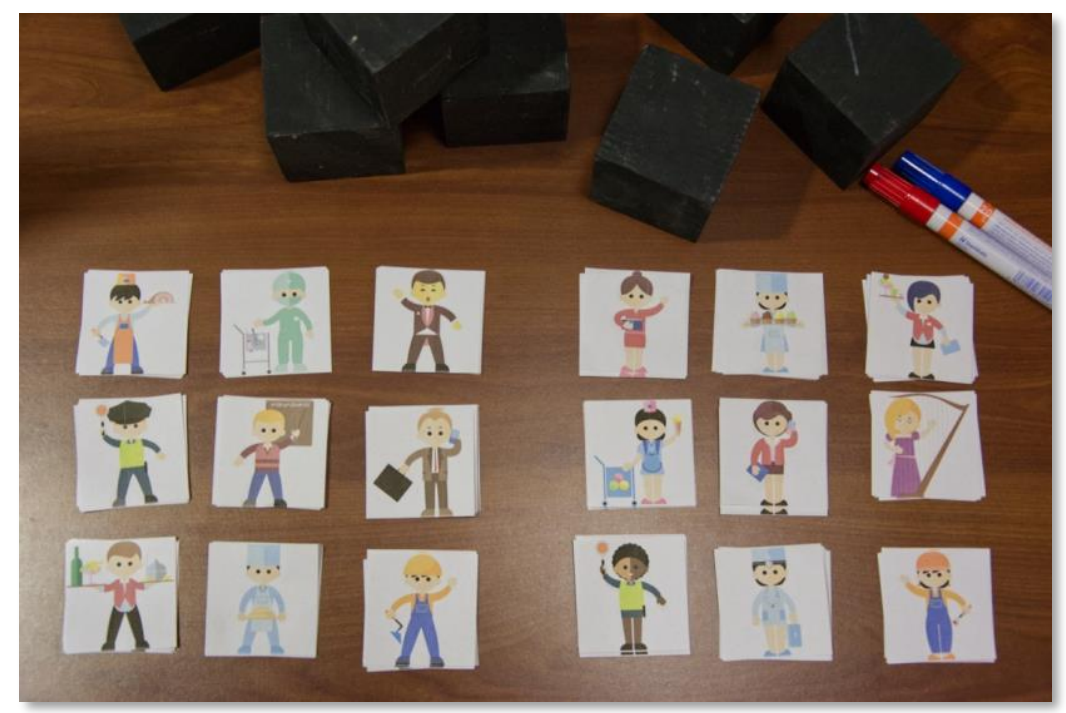


Luego, les solicita que seleccionen aquella lámina que represente mejor su interés respecto de lo que ellos desean ser cuando grandes. Posteriormente, el profesor pregunta a los estudiantes ¡cómo podemos saber cuáles son las preferencias de los niños del curso? Algunos quizás responderán que contando cuántas láminas se seleccionaron de cada profesión u oficio. Es importante que el profesor oriente la conversación para que los estudiantes de manera autónoma ideen un plan sobre cómo recolectar, organizar y representar sus respuestas (sus preferencias). Podría suceder que, en una primera instancia, los estudiantes reúnan los cubos y los agrupen de acuerdo con sus preferencias (Figura 5), o bien que los clasifiquen de acuerdo algún otro criterio.

\section{Figura 5}

Agrupando preferencias

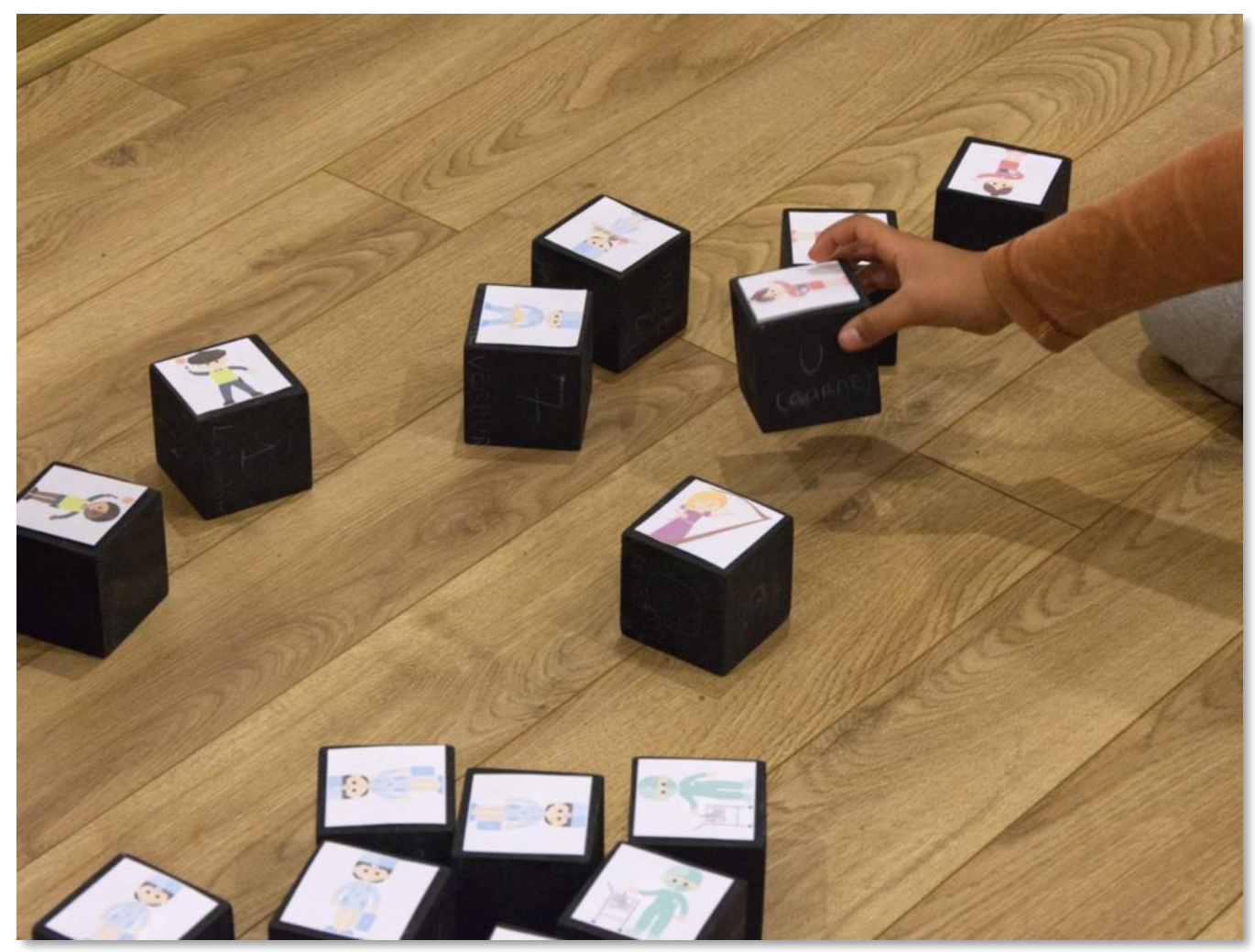

Luego, el profesor pregunta ¿cómo podemos saber cuál es la profesión u oficio más preferido por los estudiantes de este curso? Ante esto les guía para que mediante estrategias diversas identifiquen las distintas categorías, definan y apliquen distintos criterios de clasificación para organizar los datos manipulativamente, de manera de conducirles a representar estadísticamente tales datos; para luego extraer información que permita dar respuesta a la pregunta planteada. Si bien se podrán generar distintos tipos de organización, algunos podrían generar un agrupamiento vertical de los cubos por tipo de profesión u oficio (Figura 6). 


\section{Figura 6}

Primeros gráficos concretos de preferencias
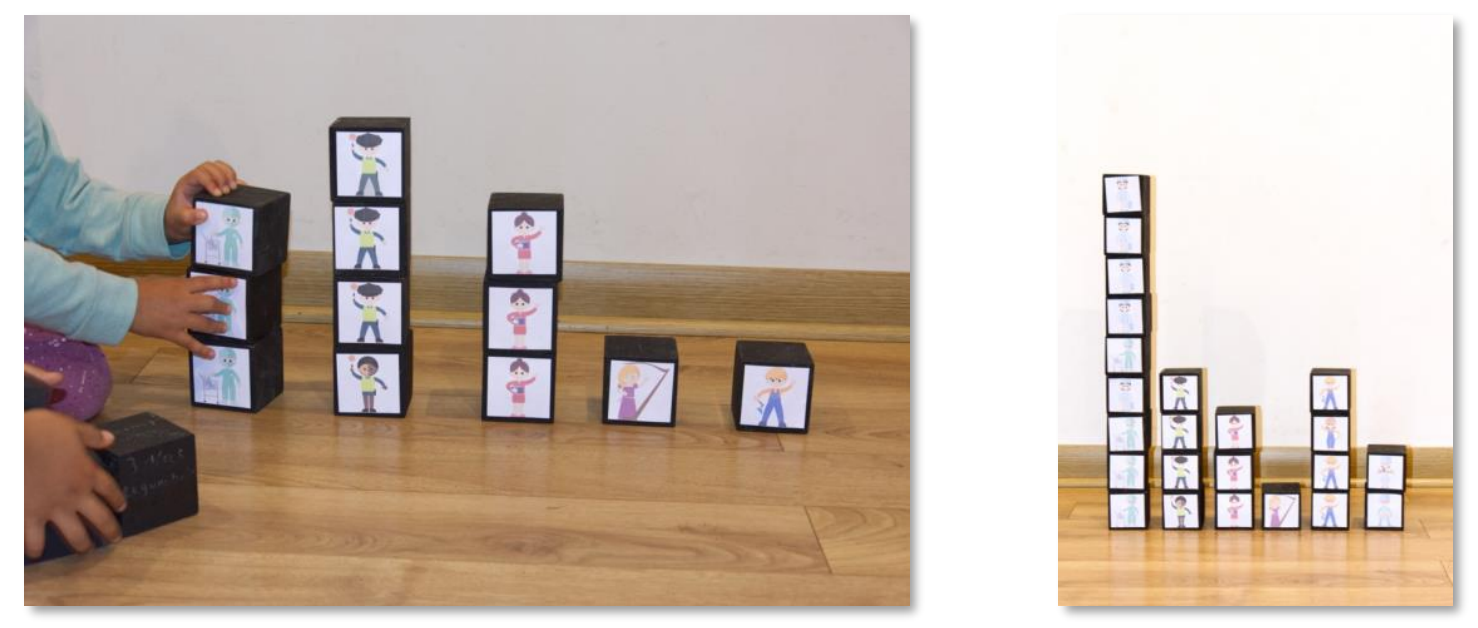

Como se observa en la Figura 6, a partir de las orientaciones del profesor, los niños agrupan y apilan los cubos según las distintas categorías de preferencias. Así, a partir de este gráfico de tipo concreto, se otorga una primera aproximación al trabajo con datos estadísticos y a una posible manera de representarlos para extraer posteriormente información. En una primera instancia, el trabajo con gráficos de tipo concreto permitirá a los estudiantes de estas edades contar con una primera aproximación a las representaciones estadísticas para más adelante transitar hacia representaciones más abstractas como, por ejemplo, los pictogramas o los gráficos de barra simple, o bien tablas de frecuencias. Igualmente, se favorece una primera aproximación a una idea intuitiva del concepto de frecuencia absoluta de una variable cualitativa, a través del recuento de los datos presentes en cada categoría. Si bien este es un concepto que no se aborda directamente en estas edades, si se encuentra vinculado al desarrollo del sentido numérico a través del conteo.

Por otro lado, es importante que el profesor plantee preguntas que permitan a los estudiantes comprender la representación de los datos, donde cada cubo representa una preferencia, favoreciendo en una primera instancia por medio de la manipulación de los datos identifiquen y definan cuáles son las distintas categorías involucradas en la variable en estudio, para luego apoyados en el conteo establezcan el recuento en cada una de ellas (frecuencia) para luego establecer comparaciones, establecer similitudes y diferencias que permitan dar respuesta a la pregunta de investigación planteada en un comienzo. Para ello, un aspecto a considerar es formular distintos tipos de preguntas que abarquen los distintos niveles de lectura de gráficos.

Asimismo, es interesante conectar la temática abordada, en este caso el tema de las profesiones $\mathrm{u}$ oficios, con el ODS de igualdad de género. Una manera de iniciar la discusión puede ser preguntando ¿existen diferencias entre las niñas y los niños respecto de lo que quieren ser cuando grandes? ¿cómo podemos saber si hay diferencias? Para dar respuesta a estos interrogantes, el profesor debe guiar a los 
estudiantes para que vean la necesidad de organizar y representar los datos de una manera distinta, que permita diferenciar entre las preferencias de las niñas y las de los niños. Una forma de abordarlo es a través de dos gráficos concretos (Figura 7) o bien a partir de un único gráfico donde una barra represente a las niñas y otra a los nińos para cada una de las categorías (Figura 8).

\section{Figura 8}

Comparando preferencias de niñas y niños a través de gráficos concretos de barra simple
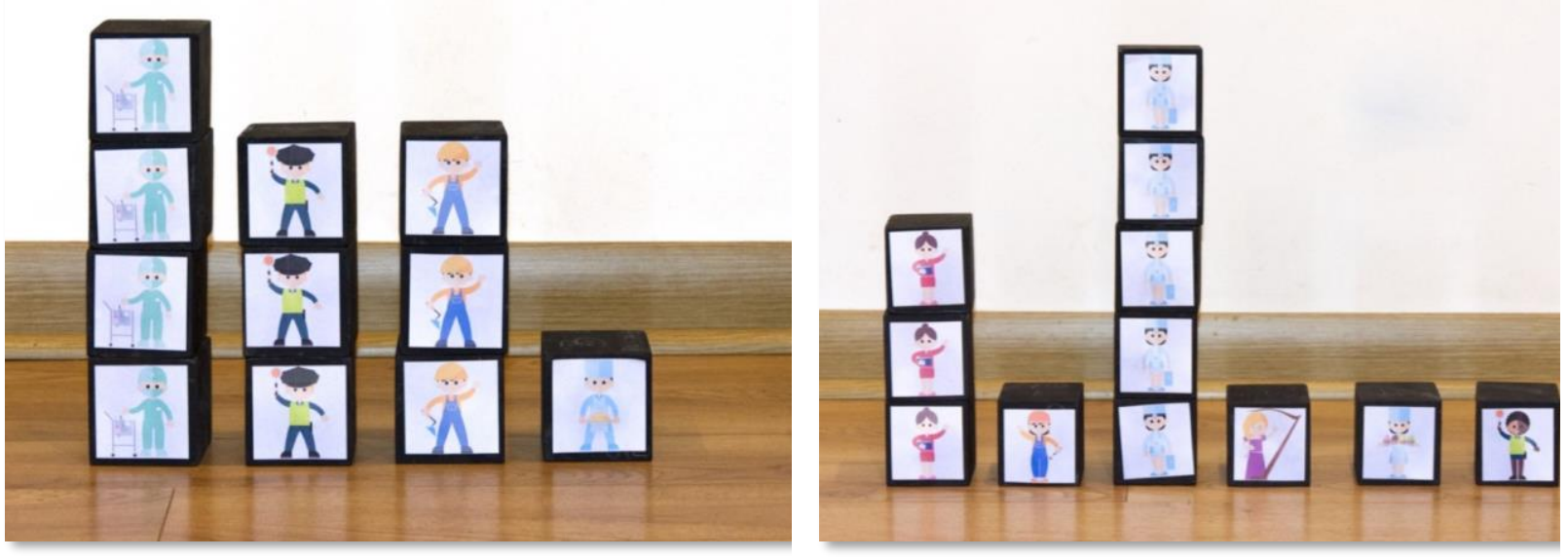

\section{Figura 8}

Comparando preferencias de niñas y niños a través de gráficos concretos de barra doble

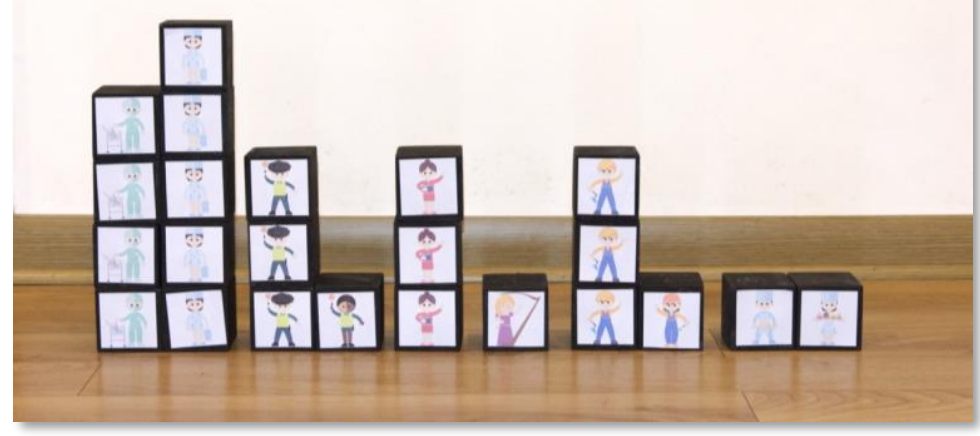

En este escenario, es conveniente que el profesor pida a los estudiantes que señalen diferencias entre las preferencias de las niñas y los niños. Seguidamente, según y cómo distribuyan los datos y la tendencia de las preferencias de profesiones u oficios, es importante que el profesor genere una discusión que lleve a impulsar el desarrollo de competencias para la sostenibilidad, por ejemplo ¿a qué creen se pueden deber estas diferencias? (pensamiento crítico); ¿consideran que existen diferencias entre los tipos 
de profesiones u oficios a los que pueden optar hombres y mujeres? (normativa); ¿cómo creen ustedes que podríamos fomentar que no existan diferencias entre las distintas profesiones u oficios a los que pueden optar hombres y mujeres? (competencia estratégica, resolución de problemas); ¿qué sucedería si solo los hombres pudieran optar a determinadas profesiones u oficios? ¿cómo afectaría a nuestro diario vivir? (pensamiento sistémico y anticipación).

Sin duda, el plantear estos tipos de preguntas, que van más allá de preguntar ¿cuántas niñas y niños prefieren cada profesión u oficio?, provocará que los estudiantes reflexionen respecto de que existen concepciones erróneas acerca de las labores que pueden realizar hombres y mujeres; que respeten y comprendan distintos tipos de opiniones (competencia de colaboración), y que finalmente, reconozcan, comprendan y cuestionen la percepción tradicional de los roles de género desde una perspectiva crítica, a la vez que respeta el anclaje cultural, donde a pesar de que hombres y mujeres son diferentes (a nivel fisiológico), todos y todas tienen a una igualdad de oportunidades.

El desarrollo de proyecto estocásticos orientados a la acción como el anterior, permitirá que los estudiantes vivencien el ciclo de investigación estadística y se aproximen al desarrollo de una alfabetización estadística, a partir del planteamiento de una problemática de interés (¿qué quieres ser cuando grande?), que implique la planeación sobre cómo recoger y manejar los datos para luego analizarlos por medio de, en este caso, representaciones gráficas y, finalmente, extraer conclusiones. Por otra parte, se favorecerá el desarrollo de distintas competencias clave para la sostenibilidad que les sirva de base para reflexionar y a cambiar su actuar y cuestionar prejuicios o ideas preconcebidas acerca de las profesiones u oficios que pueden desarrollar hombres y mujeres, afrontando así las diferencias de género, lo que contribuirá a derribar desde temprana edad algunas asociaciones erróneas de roles sexistas.

\section{EXPERIENCIA 2: TENENCIA RESPONSABLE DE MASCOTAS}

- Edad: 6-8 años.

- Contenidos implicitos: representaciones gráficas (gráficos de barra simple), frecuencia absoluta, comparación de frecuencias, variables, categorías de una variable y etapas del ciclo de investigación estadística.

- Objetivo de desarrollo sostenible: ODS 11 - Ciudades y comunidades sostenibles.

- Competencias de sostenibilidad: pensamiento sistémico, anticipación, normativa, estratégica, colaboración, pensamiento crítico, autoconciencia e integrada de resolución de problemas.

\section{DESCRIPCIÓN DE LA ACTIVIDAD}

A partir de la lectura de la noticia que se muestra en la Figura 9, el profesor introduce el tema de la tenencia responsable de mascotas, preguntando a sus alumnos acerca de si ¿̇ienen mascotas?, así como 
a partir de la lectura, ¿cuáles son las amenazas y problemáticas que se pueden desencadenar a raíz de los perros sin dueño?

\section{Figura 9}

Noticia perros sin dueño alrededor del mundo (ver https://nyti.ms/31gCKHB)

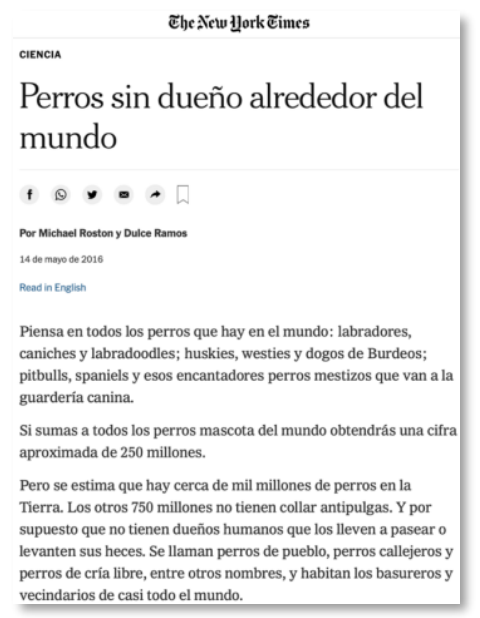

Luego de generar discusión en torno a esta problemática, el profesor presenta a sus estudiantes el gráfico de barras de la Figura 10, en el cual se muestra una estimación del número de perros callejeros en diversos países europeos. La presentación tanto de la lectura como de la gráfica tiene por propósito sensibilizar a los estudiantes con la problemática de la tenencia responsable de mascotas, los cuidados/obligaciones que ello involucra. Lo anterior con el propósito de que los estudiantes comiencen a reflexionar sobre cómo nuestras acciones pueden lograr que las ciudades y los asentamientos humanos sean inclusivos, seguros, resilientes y sostenibles.

\section{Figura 10}

La Europa de los perros sin dueño (ver https://es.statista.com)

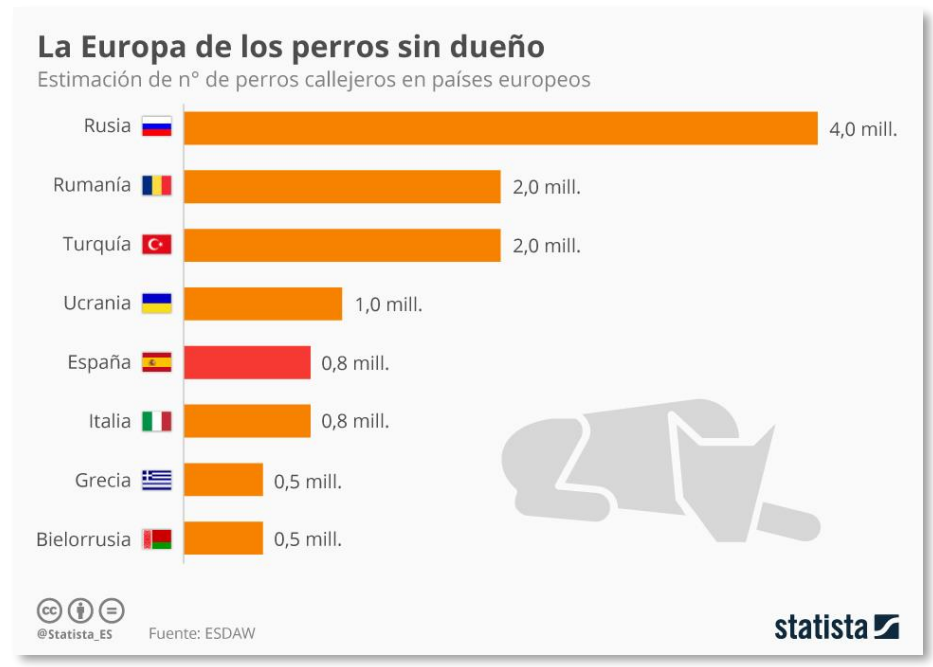


Para esto, es necesario iniciar el trabajo de análisis e interpretación de los datos presentes en la gráfica, planteando preguntas del tipo: ¿qué representa la gráfica? ¿a qué tipo de gráfico corresponde? ¿cuáles son las variables involucradas? ¿por qué?

También es importante que el docente plantee preguntas que involucre distintos niveles de lectura de gráficos, por ejemplo: ¿cuántos perros callejeros hay en España?, ¿en qué país hay un mayor número de perros callejeros?, ¿cuál es el país con un menor número de perros callejeros? Para responder a este tipo de preguntas, es necesario que los estudiantes comparen entre los datos de los distintos países, más allá de una lectura puntual de los datos. De igual manera, es importante plantear preguntas más avanzadas, que lleven a impulsar el desarrollo de competencias para la sostenibilidad como, por ejemplo: ¿creen que hay alguna razón por la cual hay tantos perros sin dueño? (pensamiento crítico), ¿qué sucederá en países latinoamericanos? (anticipación), ¿el número de perros callejeros será mayor o menor que en Europa?, ¿por qué? El plantear preguntas de este tipo tiene por propósito gatillar el interés de los estudiantes por averiguar qué sucede en su comunidad, ciudad o lugar donde viven en relación con el número de perros sin dueño. De modo tal que, a partir de estas preguntas, los estudiantes puedan desarrollar un proyecto estocástico orientado a la acción que involucre esta problemática. Para ello, es necesario orientarles en el proceso de implementar el ciclo de investigación estadística, planteado preguntas tales como: ¿cómo podemos abordar este desafío?, ¿qué datos se deben recolectar?, ¿de qué manera?, ¿cómo se registrará la información?, ¿qué tipo de representaciones estadísticas de las que conoces se pueden utilizar? Idealmente es altamente recomendable que los estudiantes aborden el desarrollo de este proyecto mediante el trabajo en equipo.

Una vez desarrollado el ciclo de investigación, es importante que los equipos de trabajo den a conocer los resultados del proyecto, tanto a sus compañeros de clase como a la comunidad. Al mismo tiempo, se les puede motivar a realizar propuestas concretas a cerca de ¿cuáles medidas se pueden tomar para disminuir el número de perros callejeros en su localidad? (estratégica, resolución de problemas y colaboración), ¿los miembros de nuestra comunidad demuestran un comportamiento responsable con la tenencia de sus mascotas? (autoconciencia y normativa), ¿qué implicaciones tendría que todos fuéramos responsables con la tenencia de nuestras mascotas? ¿cómo impactaría esto en nuestro barrio? (pensamiento sistémico y anticipación). Un punto muy importante en el desarrollo de este proyecto es que los alumnos no solo apliquen el ciclo de investigación estadística, sino que también se debe promover que puedan reflexionar críticamente sobre cuáles son sus deberes para con sus mascotas y los cuidados y responsabilidades que ello implica (competencia normativa). En definitiva, se trata de que los alumnos desarrollen un pensamiento crítico, además de otras competencias clave para la sostenibilidad, tomen autoconciencia sobre el rol que cada uno tiene en la comunidad local y en la sociedad, y sobre todo actúen, ayudando a disminuir, en este caso, el número de perros callejeros en su localidad, promoviendo una tenencia responsable de mascotas. 


\section{EXPERIENCIA 3: LA IMPORTANCIA DE BEBER AGUA}

- Edad: 8-10 años.

- Contenidos implícitos: representaciones gráficas (gráficos de líneas), frecuencia absoluta, comparación de frecuencias, variables, categorías de una variable, etapas del ciclo de investigación estadística.

- Objetivo de desarrollo sostenible: ODS 3 - Salud y Bienestar.

- Competencias de sostenibilidad: pensamiento sistémico, anticipación, normativa, estratégica, colaboración, pensamiento crítico, autoconciencia e integrada de resolución de problemas.

\section{DESCRIPCIÓN DE LA ACTIVIDAD}

Esta actividad se inicia al plantear las preguntas: ¿qué tan importante es beber agua todos los días?, ¿cuáles son los beneficios de beber agua diariamente? Así, a través de este tipo de preguntas se espera dar inicio a la discusión acerca de la importancia de beber agua, los beneficios para nuestra salud, sensibilizando a los estudiantes con esta temática que se encuentra asociada al ODS 3 salud y bienestar, que busca garantizar una vida sana y promover el bienestar para todas y todos en todas las edades. Seguidamente, el profesor muestra al estudiante el gráfico de líneas de la Figura 11. En una primera instancia, es conveniente plantear preguntas sencillas, por ejemplo, ¿de qué trata el gráfico?, ¿qué representan las líneas de cada color?, ¿qué variables se están representando? y ¿qué significado tiene el consumo de litros per cápita?

\section{Figura 11}

Consumo de bebidas no alcohólicas (ver https://www.latercera.com)

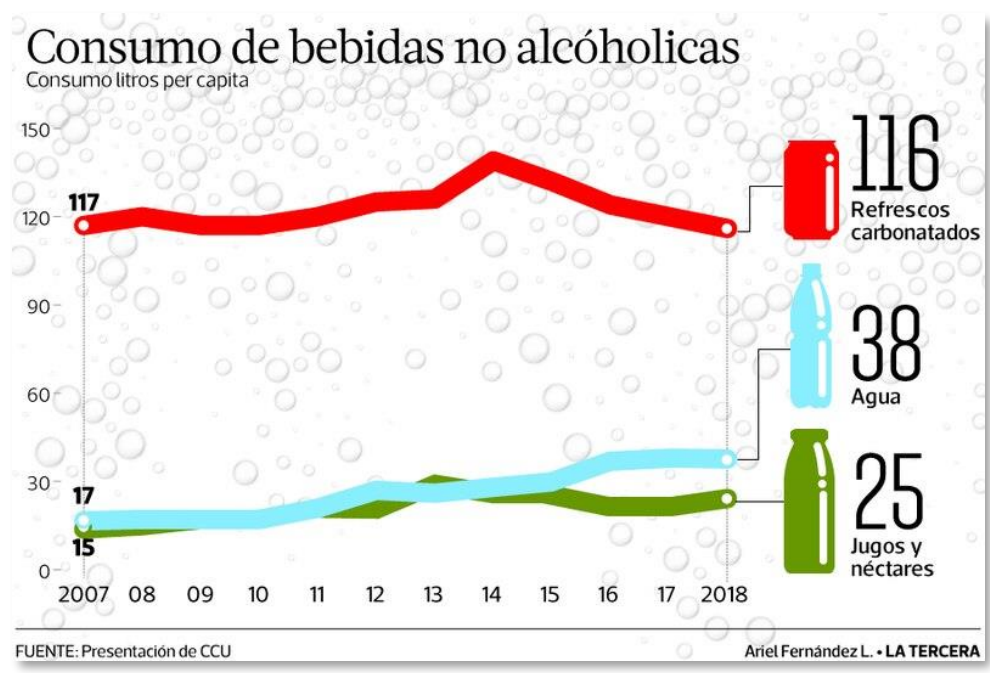

Proponer este tipo de preguntas permitirá a los estudiantes aproximarse al reconocimiento de los datos representados en la gráfica, para luego plantear preguntas un poco más elaboradas y que 
consideren los distintos niveles de lectura de gráficos, como por ejemplo: ¿qué diferencias existen entre el consumo de litros per cápita de las bebidas que se representan?, ¿qué ha ocurrido con el consumo de agua embotellada a lo largo de los años?, ¿cómo se esperaría que fuera el consumo de agua embotellada en los próximos 10 años?, ¿por qué?, ¿creen que existe alguna relación entre el alza en el consumo de agua embotellada y la disminución del consumo de refrescos carbonatados?, ¿por qué?

A través de estas preguntas se busca detonar el interés de los estudiantes por averiguar qué sucede en su entorno cercano, por ejemplo, en su comunidad educativa respecto al consumo de agua. Así, una vez concluida la etapa de lectura e interpretación gráfica, el profesor puede plantear el desafío de desarrollar un proyecto estocástico orientado a la acción, referido a ¿cuánta agua beben diariamente los estudiantes de la comunidad escolar? Cabe señalar, que en este proceso el profesor debe guiar a los estudiantes en la correcta implementación de las distintas etapas del ciclo de investigación, pues no solo se trata de implementar dicho ciclo, sino también se busca desarrollar competencias clave para la sostenibilidad que lleven a los estudiantes a reflexionar y cambiar su actuar, sus hábitos en relación con el consumo de agua diaria, reconociendo los beneficios que este tiene para su salud. En este sentido, es importante que el profesor plantee preguntas para trabajar aspectos vinculados al ODS 3 y que a la vez se planteen preguntas que fomenten competencias clave para la sostenibilidad, por ejemplo: ¿qué impacto puede tener en nuestra salud un consumo adecuado de agua? (pensamiento crítico, anticipación y sistémica), ¿nuestro consumo de agua para beber es acorde a lo recomendado para nuestra salud? (pensamiento crítico, autoconciencia y normativa), ¿cómo podríamos fomentar que los estudiantes de nuestra comunidad educativa beban mas agua? ¿qué acciones podríamos promover? (estratégica, resolución de problemas y colaboración)

\section{EXPERIENCIA 4: AVES AMENAZADAS DE CHILE}

- Edad: 10-12 años.

- Contenidos implicitos: representaciones gráficas (gráficos circulares), variables, categorías de una variable, etapas del ciclo de investigación estadística.

- Objetivo de desarrollo sostenible: ODS 13 - Acción por el Clima y ODS 15 - Vida de ecosistemas terrestres.

- Competencias de sostenibilidad: pensamiento sistémico, anticipación, normativa, estratégica, colaboración pensamiento crítico, autoconciencia, integrada de resolución de problemas.

\section{DESCRIPCIÓN DE LA ACTIVIDAD}

Por medio de esta actividad, se busca que los estudiantes tomen conciencia acerca de la importancia de proteger la avifauna que habita en el territorio nacional, cuyas especies constituyen el $5 \%$ del total de aves que existen en el mundo. Por tanto, esta actividad se encuentra orientada a sensibilizar a los estudiantes 
con el ODS 13 de acción por el clima, que busca adoptar medidas urgentes para combatir el cambio climático y sus efectos; y el ODS 15 vinculado a vida de ecosistemas terrestres, cuyo propósito es proteger, restablecer y promover el uso sostenible de los ecosistemas terrestres, gestionar sosteniblemente los bosques, luchar contra la desertificación, detener e invertir la degradación de las tierras y detener la perdida de biodiversidad.

Para ello, el profesor puede plantear la pregunta ¿qué tipo de aves conocen? A partir de esta pregunta, se debe conducir la conversación respecto de ¿cómo el uso indiscriminado de recursos naturales, la contaminación, la expansión urbana, la vulnerabilidad de los ecosistemas y la introducción de especies invasoras, entre otros aspectos, contribuyen a la perdida de la biodiversidad de la avifauna que podemos observar?

Una vez iniciada la conversación, para sensibilizar aún más a los estudiantes con el tema, el profesor puede muestra el siguiente gráfico circular o de sectores que da cuenta de las distintas especies de aves amenazadas en Chile (Figura 12).

\section{Figura 12}

Aves amenazadas de Chile (ver https://noticias.udec.cl)

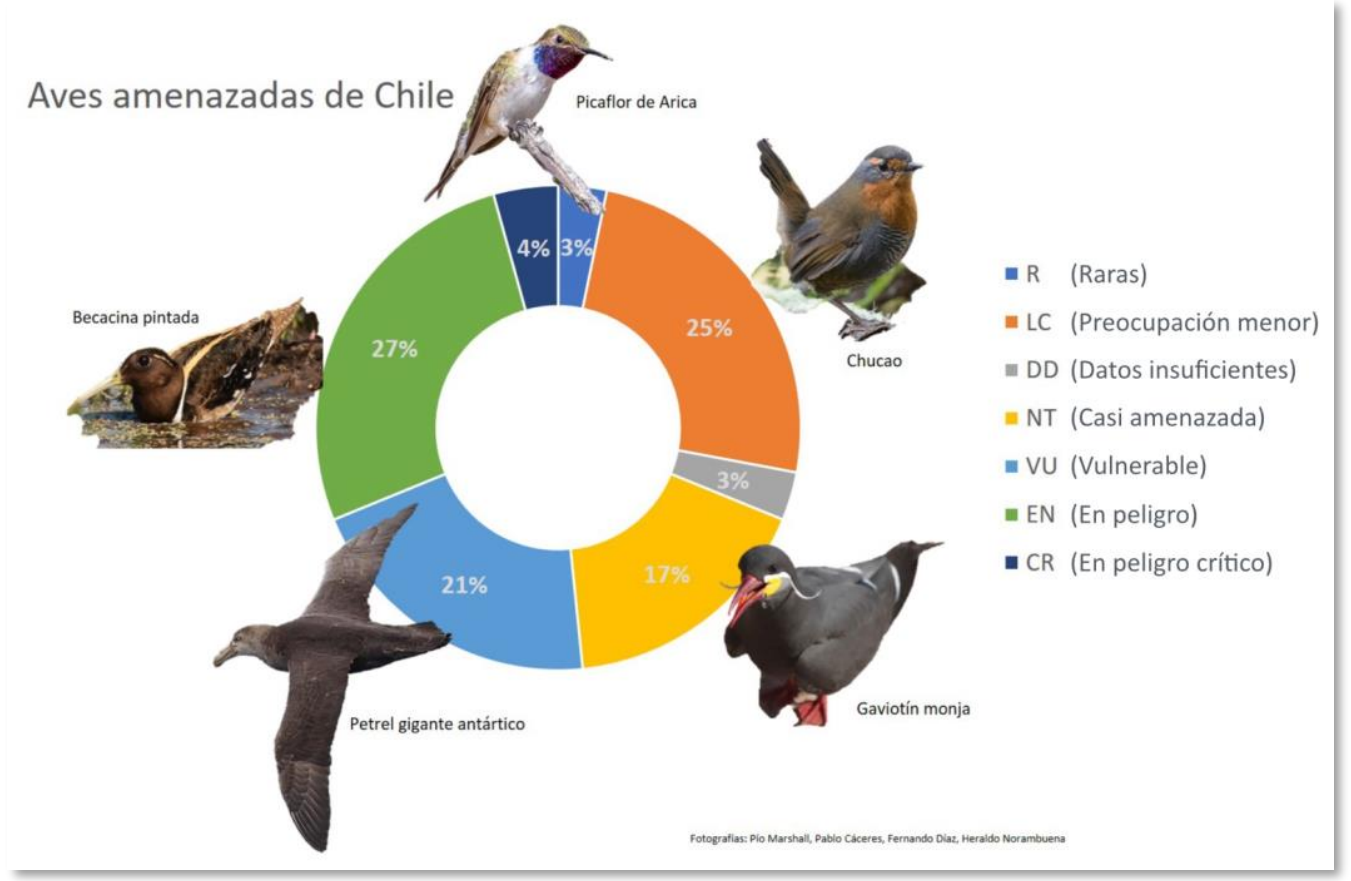

En concreto, este gráfico muestra el estado de conservación de 93 especies de aves (menos del $20 \%$ del total) de acuerdo con las categorías de: raras, preocupación menor, datos insuficientes, casi amenazada, vulnerable, en peligro y en peligro crítico. Es importante que, en una primera aproximación, el profesor plantee preguntas directas que requieren de una lectura literal de la información representada en el gráfico con el fin de comprender los datos ahí representados, por ejemplo, ¿cuál es la variable que 
se está representando?, ¿qué significa cada uno de los colores del gráfico?, ¿qué representan estos colores?, ¿qué porcentaje de especies aves se encuentra "en peligro crítico"? Para luego, comenzar a plantear preguntas con un nivel de lectura de complejidad un poco mayor y que requieren en cierta medida de un pensamiento crítico, tales como ¿cuántas especies de aves se encuentran "en peligro"? ¿qué porcentaje de especies de aves se encuentran en categorías de amenaza?

Finalmente, se pueden plantear preguntas que fomenten competencias clave para la sostenibilidad y que lleven a reflexionar, como, por ejemplo, ¿qué medidas se pueden tomar para mitigar la pérdida de biodiversidad en Chile? (normativa, estratégica, resolución de problemas, pensamiento crítico) y ¿cómo podemos contribuir para proteger la avifauna que habita en nuestra ciudad? (estratégica, colaboración, resolución de problemas).

Así, a partir de estas preguntas el profesor puede plantear el desarrollo de un proyecto estocástico orientado a la acción, en el cual, en primer lugar, los estudiantes deban indagar acerca de ¡cuáles son las especies de aves que viven en su ciudad? Guiados por el profesor, los estudiantes organizarían la implementación del ciclo de investigación y podrían, por ejemplo, visitar un parque o una plaza de la ciudad y recoger datos sobre las especies que se avistan, de modo a levantar un reporte sobre, cuáles son las especies más frecuentes, y cómo estás se relacionan con las especies que se deberían avistar. En el desarrollo de dicho proyecto se debe velar que los estudiantes vivencien las etapas del ciclo de investigación, recolectando y analizando información, prestando atención a aspectos centrales, como el planteamiento de preguntas de investigación estadística, método de recogida de datos, reconocer la variabilidad de los datos, organizar y representar los datos de manera adecuada, interpretar los datos para dar respuesta a la pregunta que guía el ciclo de investigación, así como destacar la importancia de volver a los datos y llevar a los estudiantes que vayan más allá y reflexionen a partir de ellos.

Así, una vez desarrollado el ciclo de investigación, es importante que los equipos de trabajo colaborativo den a conocer sus resultados, tanto a sus compañeros de clase como a la comunidad, mediante un video en las redes sociales o un póster, por ejemplo. Al mismo tiempo, se les puede motivar a realizar propuestas concretas acerca de cómo proteger los hábitats de las aves de su localidad o ciudad.

\section{CONSIDERACIONES FINALES}

En este artículo se presenta un conjunto de experiencias orientadas a promover competencias clave para la sostenibilidad a partir del trabajo con proyectos estocásticos que abordan problemáticas diversas provenientes de los distintos ODS. Para ello, en primer lugar, se ha descrito el vínculo entre los proyectos estocásticos orientados a la acción y la educación para la sostenibilidad, pues la Educación Estocástica es un conocimiento que se alinea perfectamente con la EDS. Por consiguiente, de acuerdo con Vásquez (2020), es imperativo avanzar hacia una Educación Estocástica para educar en sostenibilidad, enfocada en el abordaje de problemáticas provenientes de contextos reales y locales 
vinculados con los ODS, con el propósito de empoderar a los estudiantes para reflexionar sobre cuestiones vinculadas al desarrollo sostenible.

En la propuesta de experiencias de aprendizaje que se ha presentado se sigue este enfoque, con el propósito de orientar a los docentes que se desempeñan en la etapa de infantil y los primeros años de primaria en el diseño de propuestas auténticas, contextualizadas en sostenibilidad. Pues, aún existe escasa tradición de abordar estos temas en las primeras edades, pese a que diversos autores y organismos internacionales enfatizan sobre la importancia de iniciar la enseñanza de la estadística y la probabilidad desde temprana edad (Bargagliotti et al., 2020; NCTM, 2000; Vásquez et al., 2018). Por tanto, es necesario ofrecer al profesorado herramientas que les permita desarrollar en los alumnos la motivación y capacidad para comprender, interpretar, evaluar críticamente y, cuando sea pertinente, expresar opiniones en cuanto a mensajes e información cuantitativa y estadística, además de potenciar argumentos basados en datos o cuestiones relacionadas con la incertidumbre y el riesgo del mundo real (Gal, 2002, 2005), que lleve a los estudiantes, en el largo plazo, a una toma de decisiones conscientes para crear un mundo más sostenible. Más aún si consideramos que gran parte de las competencias para el desarrollo sostenible requieren de una alfabetización estadística y probabilística para su desarrollo (Vásquez, 2020).

En este contexto, los proyectos estocásticos orientados a la acción desempeñan un rol fundamental para generar instancias que permitan aprender estadística y probabilidad con sentido, en contextos de sostenibilidad. Desde este prisma, y de acuerdo con Vásquez (2021), el uso de gráficos estadísticos, así como la comprensión gráfica, se constituyen en una herramienta para promover competencias clave para la sostenibilidad, tendiendo un puente entre la alfabetización estadística y probabilística y la alfabetización en sostenibilidad.

En futuros estudios será necesario analizar la implementación de estas experiencias de aprendizaje con foco en sostenibilidad, con el propósito de evidenciar su potencial, así como aquellos aspectos que requieran de mejoras.

\section{ACLARATORIAS}

La autora no tiene conflicto de interés que declarar. El artículo ha sido realizado en el marco del proyecto FONDECYT № 1200356, financiado por la Agencia Nacional de Investigación y Desarrollo del Gobierno de Chile.

\section{REFERENCIAS}

Alsina, Á. (2017). Contextos y propuestas para la enseñanza de la estadística y la probabilidad en Educación Infantil: un itinerario didáctico. Revista Epsilon, 34(95), 25-48. 
Alsina, Á., \& Calabuig, M. T. (2019). Vinculando Educación Matemática y sostenibilidad: implicaciones para la formación inicial de maestros como herramienta de transformación social. Revista de Educación Ambiental y Sostenibilidad, 1(1), 1203. https://doi.org/10.25267/Rev educ ambient sostenibilidad.2019.v1.i1.1203

Alsina, Á., \& Mulà, I. (2019). Advancing towards a transformational professional competence model through reflective learning and sustainability: the case of mathematics teacher education. Sustainability, 11(15), 4039. https://doi.org/10.3390/su11154039

Anasagasti, J., \& Berciano, A. (2016). El aprendizaje de la estadística a través de PBL con futuros profesores de primaria. Contextos Educativos, 1(extraordinario), 31-43. https://doi.org/10.18172/con.2699

Arteaga, P., Batanero, C., Cañadas, G., \& Contreras, J. M. (2011). Las tablas y gráficos estadísticos como objetos culturales. Números, 76, 55-67.

Arteaga, P., Batanero, C., Contreras, J. M., \& Cañadas, G. (2016). Evaluación de errores en la construcción de gráficos estadísticos elementales por futuros profesores. Revista Latinoamericana de Investigación en Matemática Educativa, 19(1), 15-40. https://doi.org/10.12802/relime.13.1911

Bargagliotti, B., Franklin, C., Arnold, P., Gould, R., Jhonson, R., Perez, L., \& Spangles, D. A. (Eds.). (2020). Pre-K-12 guidelines for assessment and instruction in statistics education II (GAISE II). American Statistical Association.

Batanero, C. (2019). Treinta años de investigación en Educación Estocástica: reflexiones y desafíos. En J. M. Contreras, M. M. Gea, M. M. López-Martín, \& E. Molina-Portillo (Eds.), Actas del Tercer Congreso Internacional Virtual de Educación Estadistica (pp. 1-15). Universidad de Granada.

Batanero, C., \& Díaz, C. (Eds.). (2011). Estadistica con proyectos. Departamento de Didáctica de la Matemática.

Batanero, C., Díaz, C., Contreras, J. M., \& Roa, R. (2013). El sentido estadístico y su desarrollo. Números, 83, 7-18.

Burrill, G., \& Biehler, R. (2011). Fundamental statistical ideas in the school curriculum and in training teachers. En C. Batanero, G. Burrill, \& C. Reading (Eds.), Teaching statistics in school mathematics. Challenges for teaching and teacher education. A joint ICMI/IASE study (pp. 57-69). Springer. https://doi.org/10.1007/978-94-007-1131-0 10 
PROYECTOS ESTOCÁSTICOS ORIENTADOS A LA ACCIÓN: UNA PUERTA AL DESARROLLO...

Casas, A., Torres, I., Delgado-Lemus, A., Rangel-Lando, S., Ilsley, C., Torres-Guevara, J., Cruz, A., Parra, F., Moreno-Calles, A., Camou, A., Castillo, A., Ayala-Orozco, B., Blancos, J., Vallejo, M., Solís, L., Bullen, A., Ortiz, T., \& Frarfán, B. (2017). Ciencia para la sustentabilidad: investigación, educación y procesos participativos. Revista Mexicana de Biodiversidad, 88, 113-128.

https://doi.org/10.1016/j.rmb.2017.10.003

Cobb, G., \& Moore, D. (1997). Mathematics, statistics, and teaching. American Mathematical Montbly, 104(9), 801-823. https://doi.org/10.1080/00029890.1997.11990723

Comisión Mundial del Medio Ambiente y del Desarrollo (1987). CMMAD. Nuestro futuro común. Alianza.

Curcio, F. R. (1987). Comprehension of mathematical relationships expressed in graphs. Journal for Research in Mathematics Education, 18(5), 382-393.

https://doi.org/10.5951/jresematheduc.18.5.0382

delMas, R. C. (2004). A Comparison of mathematical and statistical reasoning. En D. Ben-Zvi, \& J. Garfield (Eds.), The challenge of developing statistical literacy, reasoning and thinking (pp. 7996). Springer. https://doi.org/10.1007/1-4020-2278-6 4

Friel, S., Curcio, F., \& Bright, G. (2001). Making sense of graphs: critical factors influencing comprehension and instructional implications. Journal for Research in Mathematics Education, 32(2), 124-158. https://doi.org/10.2307/749671

Gal, I. (2002). Adults' statistical literacy: meanings, components, responsibilities. International Statistical Review, 70(1), 1-25. https://doi.org/10.1111/j.1751-5823.2002.tb00336.x

Gal, I. (2005). Towards 'probability literacy' for all citizens. En G. Jones (Ed.), Exploring probability in school: challenges for teaching and learning (pp. 43-71). Kluwer Academic Publishers.

Gal, I. (2019). Understanding statistical literacy: about knowledge of contexts and models. En J. M. Contreras, M. M. Gea, M. M. López-Martín, \& E. Molina-Portillo (Eds.), Actas del Tercer Congreso International Virtual de Educación Estadistica (pp. 1-15). Universidad de Granada.

Gattuso, L., \& Ottaviani, M. G. (2011). Complementing mathematical thinking and statistical thinking in school mathematics. En C. Batanero, G. Burrill, \& C. Reading (Eds.), Teaching statistics in school mathematics-challenges for teaching and teacher education: a joint ICMI/IASE study (pp. 121-132). Springer. https://doi.org/10.1007/978-94-007-1131-0

González, M. T., Espinel, M. C., \& Ainley, J. (2011). Teachers' graphical competence. En C. Batanero, G. Burrill, \& C. Reading (Eds.), Teaching Statistics in School Mathematics Challenges for 
Teaching and Teacher Education: a joint ICMI IASE Study (pp. 187-197). Springer. https://doi.org/10.1007/978-94-007-1131-0 20

Hahn, C. (2014). Linking academic knowledge and professional experience in using statistics: a design experiment for business school students. Educational Studies in Mathematics, 86(2), 239-251. https://doi.org/10.1007/s10649-011-9363-9

Inzunza, S. (2017). Potencial de los proyectos para desarrollar motivación, competencias de razonamiento y pensamiento estadístico. Actualidades investigativas en educación, 17(3), 1-30. https://doi.org/10.15517/AIE.V1713.29874

Joutsenlahti, J., \& Perkkilä, P. (2019). Sustainability development in Mathematics Education - A case study of what kind of meanings do prospective class teachers find for the mathematical symbol “2/3”? Sustainability, 11(2), 457. https://doi.org/10.3390/su11020457

Leicht, A., Heiss, J., \& Byun, W. J. (2018). Issues and Trends in Education for Sustainable Development. UNESCO Publishing.

NCTM. (2000). Principles and standards for school mathematics. The National Council of Teachers of Mathematics.

Novo, M. L., Cuida, A., \& Encinas, M. (2020). Un acercamiento a la sostenibilidad desde la Educación Matemática Realista en el aula de Infantil. Edma 0-6: Educación Matemática en la Infancia, 9(2), 37-50.

OCDE. (2019). OECD Future of education and skills 2030: OECD learning compass 2030. OECD.

Rychen, D. S. (2003). Competencias clave: abordar desafíos importantes en vida. En D. S. Rychen, \& L. H. Salganik (Eds.), Competencias clave para una vida exitosa y una sociedad sana (pp. 63-107). Hogrefe \& Huber Publishers.

Shaughnessy, J. M., Garfield, J., \& Greer, B. (1996). Data handling. En A. J. Bishop, K. Clements, C. Keitel, J. Kilpatrick, \& C. Laborde (Eds.), International Handbook of Mathematics Education (pp. 205-237). Kluwer. https://doi.org/10.1007/978-94-009-1465-0_7

Stibbe, A. (2009). The Handbook of Sustainability Literacy: Skills for a Changing World. Green Books.

UNESCO. (2015). Transformar nuestro mundo: la Agenda 2030 para el desarrollo sostenible.

Organización de las Naciones Unidas para la Educación, la Ciencia y la Cultura. 
UNESCO. (2017). Educación para los objetivos de desarrollo sostenible: objetivos de aprendizaje.

Organización de las Naciones Unidas para la Educación, la Ciencia y la Cultura.

Vásquez, C. (2020). Educación Estocástica: una herramienta para formar ciudadanos de sostenibilidad. Matemáticas, Educación y Sociedad, 3(2), 1-20. http://hdl.handle.net/10396/20628

Vásquez, C. (2021). Comprensión y uso docente de gráficos estadísticos por futuros profesores para promover competencias para la sostenibilidad. PARADIGMA, 41(e1), 165-190. https://doi.org/10.37618/PARADIGMA.1011-2251.2021.p165-190.id1022

Vásquez, C., \& Alsina, Á. (2021). Conectando educación estadística y educación para la sostenibilidad: un marco para promover el desarrollo sostenible en la formación del profesorado. En T. Sola, S. Alonso, M. G. Fernández, \& J. C. De La Cruz (Eds.), Estudios sobre Innovación e Investigación Educativa (pp. 973-985). Editorial DYKINSON, S. L.

Vásquez, C., \& García-Alonso, I. (2020). La educación estadística para el desarrollo sostenible en la formación del profesorado. Profesorado. Curriculum y formación del profesorado, 24(3), 125-147. https://doi.org/10.30827/profesorado.v24i3.15214

Vásquez, C., Coronata, C., \& Rivas, H. (2021). Enseñanza de la estadística y la probabilidad de los 4 a los 8 años de edad: una aproximación desde los procesos matemáticos en libros de texto chilenos. PNA, 15(4), 339-365. https://doi.org/10.30827/pna.v15i4.22512

Vásquez, C., Díaz-Levicoy, D., Coronata, C., \& Alsina, Á. (2018). Alfabetización estadística y probabilística: primeros pasos para su desarrollo desde la Educación Infantil. Cadernos Cenpec, 8(1), 154-179. http://dx.doi.org/10.18676/cadernoscenpec.v8i1.393

Vásquez, C., García-Alonso, I., Seckel, M. J., \& Alsina, Á. (2021). Education for sustainable development in primary education textbooks - An educational approach from statistical and probabilistic literacy. Sustainability, 13(6), 3115. https://doi.org/10.3390/su13063115

Vásquez, C., Pincheira, N., Piñeiro, J. L., \& Díaz-Levicoy, D. (2019). ¿Cómo se promueve el aprendizaje de la estadística y la probabilidad? Un análisis desde los libros de texto para la Educación Primaria. BOLEMA, 33(65), 1133-1154. https://doi.org/10.1590/1980-4415v33n65a08

Wals, A. E. J. (2015). Más allá de dudas no razonables. Educación y aprendizaje para la sostenibilidad socioecológica en el Antropoceno. Universidad de Wageningen. 
Weiland, T. (2019). The contextualized situations constructed for the use of statistics by school mathematics textbooks. Statistics Education Research Journal, 18(2), 18-38.

https://doi.org/10.52041/serj.v18i2.138

Wells, H. G. (1911). Mankind in the making (5. ${ }^{a}$ ed.). Chapman and Hall (original publicado en 1903).

WHO. (2020). Munich Security Conference. World Health Organization webpage. https://www.who.int/director-general/speeches/detail/munich-security-conference

Wild, C. J., \& Pfannkuch, M. (1999). Statistical thinking in empirical enquiry. International Statistical Review, 67(3), 223-248. http://dx.doi.org/10.1111/j.1751-5823.1999.tb00442.x

Yurovsky, D., Boyer, T., Smith, L. B., \& Yu, C. (2013). Probabilistic cue combination: less is more. Developmental Science, 16(2), 149-158. https://doi.org/10.1111/desc.12011

\section{Cómo citar este artículo:}

Vásquez, C. (2021). Proyectos estocásticos orientados a la acción: una puerta al desarrollo sostenible desde temprana edad. Revista Venezolana de Investigación en Educación Matemática (REVIEM), 1(2), e202108. https://doi.org/10.54541/reviem.v1i2.10 
PROYECTOS ESTOCÁSTICOS ORIENTADOS A LA ACCIÓN: UNA PUERTA AL DESARROLLO...

(c) () Copyright (c) 2021. Claudia, Vásquez Esta obra está protegida por una licencia Creative Commons 4.0. International (CC BY 4.0).

Usted es libre para Compartir — copiar y redistribuir el material en cualquier medio o formato - y Adaptar el documento - remezclar, transformar y crear a partir del material — para cualquier propósito, incluso para fines comerciales, siempre que cumpla la condición de:

Atribución: Usted debe dar crédito a la obra original de manera adecuada, proporcionar un enlace a la licencia, e indicar si se han realizado cambios. Puede hacerlo en cualquier forma razonable, pero no de forma tal que sugiera que tiene el apoyo del licenciante o lo recibe por el uso que hace de la obra.

$\underline{\text { Resumen de licencia - Texto completo de la licencia }}$ 